\title{
Diagnostic Variations and Treatment Responses of Catatonic Patients in the Psychiatric In-Patient Department of a Rural Tertiary Care Centre - A Clinical Study from Eastern India
}

\author{
Uttam Majumder ${ }^{1}$, Avik Kumar Layek ${ }^{2}$ \\ ${ }^{1}$ Department of Psychiatry, North Bengal Medical College, Siliguri, West Bengal, India. \\ ${ }^{2}$ Department of Psychiatry, North Bengal Medical College, Siliguri, West Bengal, India.
}

\section{ABSTRACT}

\section{BACKGROUND}

There are very few studies regarding diagnostic variations and treatment response in catatonia in India. We wanted to assess the treatment response according to the background psychiatric diagnoses of in-patient catatonic patients.

\section{METHODS}

This is a cross-sectional descriptive study done in the Psychiatric indoor of North Bengal Medical College between December 2018 to December 2019. Catatonia was diagnosed using DSM- 5 criteria in the patients admitted. A total 30 patients were included in the study. The patients were assessed by Bush-Francis Catatonia Rating Scale, were given oral or parenteral lorazepam as needed and observed for 2-3 weeks. Non-responders were given modified ECT as per convention. Background diagnoses were checked by thorough history taking and investigations.

\section{RESULTS}

Majority of the patients were Hindu males in their twenties from rural lower-middle socio-economic families, around $20 \%$ of them having family history of affective and psychotic illnesses. They were diagnosed mostly with schizophrenia and other psychotic illnesses (53\%) and mood disorders (30\%). The mean BFCRS score at the time admission was $20.93 \pm 6.16$. The commonly found symptoms were mutism, staring, negativism, rigidity, posturing/catalepsy, mannerism, and withdrawal. It was seen that $83.33 \%$ of cases responded well to lorazepam only, whereas the rest $13.33 \%$ required ECT after non-response or partial response to lorazepam. Only one case after diagnosis with catatonia following organic condition was referred to the Medicine Department. The duration of hospital stay was found to be significantly different [F= $3.58(>3.35)]$ among different diagnoses groups (mood disorders, psychotic disorders and others) when the catatonic severity, lorazepam dosage and the duration of treatment response were assessed among those groups using multiple one-way ANOVA.

\section{CONCLUSIONS}

This study has yielded important findings regarding the age and socio-demographic profiles of the patients, family history of psychiatric disorders, clinical features, and significance of diagnostic variations in relation to the treatment with lorazepam and ECT in catatonic patients in the psychiatric indoor in a tertiary care rural hospital of Eastern India.

\section{KEY WORDS}

Catatonia, BFCRS, Lorazepam, Negative Symptoms
Corresponding Author: Dr. Avik Kumar Layek, Flat No. 1, Bharati Apartment, Thiknikata, Sushrutanagar, Darjeeling-734012, West Bengal, India.

E-mail:avik.layek@gmail.com

DOI: $10.14260 /$ jemds/2020/165

Financial or Other Competing Interests: None.

How to Cite This Article:

Majumder $U$, Layek AK. Diagnostic variations and treatment responses of catatonic patients in the psychiatric inpatient department of a rural tertiary care centre- a clinical study from Eastern India. J. Evolution Med. Dent. Sci. 2020;9(10): 759-763, DOI: 10.14260/jemds/2020/165

Submission 19-12-2019,

Peer Review 16-02-2020,

Acceptance 22-02-2020,

Published 09-03-2020. 


\section{BACKGROUND}

Catatonia, a term first coined by K L Kahlbaum is a symptom complex comprising of motor abnormality along with disorders of mood, behaviour or thought. ${ }^{1}$ He told about 17 motor abnormalities seen in patients of disorders of mood and thought, tuberculosis, and epilepsy. Later Emile Kraepelin mentioned catatonic state in chronic mentally ill patients including that of dementia praecox. ${ }^{2}$ It happens to occur as a presenting complaint in $7-15 \%$ of acute psychiatric inpatients. ${ }^{3}$ Around 40 signs have been described relating to catatonia over the years having overlapping definitions. ${ }^{4}$ Those might be of hypokinetic, stuporous or hyperkinetic, along with posturing, stereotypies and mannerisms. Sometimes there may be mutism, echoing words and phrases or verbigeration, negativism, automatic obedience, flexibility, mitgehen, gegenhalten. These signs can present in retardedstuporous type or excited-delirious type. ${ }^{4}$ As per DSM-5, the presence of three prominent signs for at least $24 \mathrm{~h}$ is suffices for catatonia diagnosis. ${ }^{3}$

Catatonia is present in both acute and chronic psychiatric patients. It is difficult to study, understand and recognize catatonia though it is pretty common. It actually challenges the clinician's diagnostic and management skills. The presentation remains unclear owing to many overlapping domains rendering the theoretical approach to the scenario debatable.5,6 It has been observed that catatonic signs were present in nearly $20-40 \%$ of in-patients diagnosed with mood and psychotic disorders studies of Indian settings.7-10 regarding the background diagnoses, most Indian studies, unlike that of western ones, show a higher contribution of psychotic illnesses rather than mood disorders producing catatonic symptoms. $7,11,12$

Prior Indian studies mostly yielded mutism, immobility/stupor, staring, negativism, rigidity, posturing/catalepsy and withdrawal as the catatonic phenomenology.7,11,12 Characteristically the treatment response and background diagnoses of catatonia were very sparsely dealt with in prior Indian studies. ${ }^{11}$ The wide variety of catatonic signs and symptoms resulted in the inception of a few scales for identification and severity assessment like Bush-Francis Catatonia Rating Scale (BFCRS) ${ }^{13}$ and the modified Roger's Rating Scale.14,15 The quick resolution of symptoms by lorazepam challenge test often indirectly help in the assessment of catatonia though this may happen often temporarily. ${ }^{16}$ Catatonia of various forms can be managed with benzodiazepines, specially lorazepam. Although lorazepam succeeds in treating catatonia with fair results, different patients may respond in a varying manner. ${ }^{17-20}$ For partial responses and prolonged catatonia, ECT can be applied.10 Drugs acting on NMDA receptors, e.g. memantine have also showed to have promising future in the treatment of catatonia. ${ }^{21}$

There are only a few studies that have been conducted in India with the aim to explore the background diagnoses and treatment response in patients presenting with catatonia in inpatient treatment settings. ${ }^{10,11}$ As there is scarcity of related literature specially in the eastern part of India, this study was conducted to assess the response to lorazepam and the duration among various diagnostic categories having catatonic syndrome in this part of the country.

\section{METHODS}

North Bengal Medical College, established in 1968 in the northern part of West Bengal, an eminent centre providing tertiary care and multidisciplinary postgraduate education, is situated in the gateway of North-East India with inpatient and outpatient facilities. The Psychiatry department with daily OPD Attendance of roughly 300 patients and Inpatient bed strength of 40 offers patient care and post graduate training. It was a cross-sectional descriptive study with purposive sampling. In our study, after obtaining the clearance from the Institution Ethics Committee and taking the informed consent, all patients admitted in the indoor who were diagnosed with catatonia by two faculty psychiatrists as per DSM-5 criteria 3 , were routinely assessed using a detailed semi-structured data sheet. All the patients admitted with catatonia during last one year (December 2018 to December 2019) were consecutively taken for the study barring those who did not consent and those with organic presentation at admission. Bush Francis Catatonic Rating scale was applied for evaluation to monitor the response to treatment.

The Bush-Francis Scale, ${ }^{13}$ created in 1996, is the most widely used due to its validity, reliability and ease of application. The Bush-Francis Scale has two versions: a longer one (BFCRS) with 23 items rated from 0 to 3 to evaluate catatonic symptom severity; and a reduced version, the Bush-Francis Catatonia Screening Instrument (BFCSI), with only the first 14 items, to evaluate presence or absence of catatonic symptoms, and to screen for syndrome. In the present study the longer version is used. After detailed evaluation, all patients presenting with catatonia are initially given a trial of parenteral or oral lorazepam for 1-2 weeks at doses ranging from $4-16 \mathrm{mg} /$ day as per the existing treatment protocol of catatonia and as reciprocated by multiple prior studies.4,7,11,12,17 Longer duration was considered in case of slower response in few cases. Patients who do not respond to lorazepam trial are applied modified electroconvulsive therapy (MECT) as per the standard treatment protocol.7,22 Once they recover from catatonia they are evaluated for lifetime psychiatric illnesses as per DSM- 5 and treated with antipsychotics and/or mood stabilizers as per the existing guideline maintained in the in-patient department.

\section{Statistical Analysis}

The data obtained were collected in Microsoft Office Excel Worksheet. Means and ranges were calculated in terms of socio-demographic and clinical information. Analysis of variance (ANOVA) was done using the Statistical Package for the Social Sciences (SPSS version 22) where the obtained variances in particular variables could be classified into sections owing to diverse sources of variation.

\section{RESULTS}

During the given period of 1 year, a total of 30 patients were taken for the study with provisional diagnosis of catatonia, the mean age of presentation of catatonia being 24.63 years. 
Prevalence was more in males (63.3\%), mostly (43.3\%) with high school education, Hindu religion (76.7\%), mostly from rural background (70\%), majority belonging to semiskilled worker $(30 \%)$ and housewife $(23.3 \%)$ by occupation, mostly from lower middle socio-economic status (56.7\%) as per the Modified Kuppuswamy scale as shown in Table 1.

\begin{tabular}{|c|c|c|c|c|c|c|c|c|c|}
\hline \multirow[t]{2}{*}{ Age } & \multicolumn{3}{|c|}{$<20$ Years } & \multicolumn{3}{|c|}{ 21-30 Yrs. } & \multicolumn{3}{|c|}{$>30$ Years } \\
\hline & \multicolumn{3}{|c|}{7} & \multicolumn{3}{|c|}{17} & \multicolumn{3}{|c|}{6} \\
\hline \multirow{2}{*}{ Gender } & \multicolumn{3}{|c|}{ Male } & \multicolumn{3}{|c|}{ Female } & \multicolumn{3}{|c|}{ Others } \\
\hline & \multicolumn{3}{|c|}{19} & \multicolumn{3}{|c|}{11} & \multicolumn{3}{|c|}{0} \\
\hline \multirow{2}{*}{ Religion } & \multicolumn{3}{|c|}{ Hindu } & \multicolumn{3}{|c|}{$\begin{array}{c}\text { Muslim } \\
5\end{array}$} & \multicolumn{3}{|c|}{ Others } \\
\hline & \multicolumn{3}{|c|}{23} & \multirow{2}{*}{\multicolumn{3}{|c|}{5}} & \multicolumn{3}{|c|}{2} \\
\hline \multirow{2}{*}{ Background } & \multirow{2}{*}{\multicolumn{6}{|c|}{$\frac{\text { Urban }}{9}$}} & \multicolumn{3}{|c|}{ Rural } \\
\hline & & & & & & & & 21 & \\
\hline \multirow[t]{2}{*}{ 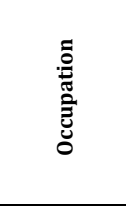 } & $\begin{array}{l}\stackrel{n}{0} \\
\frac{\tilde{D}}{\tilde{E}} \\
\dot{\omega}\end{array}$ & 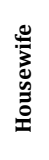 & 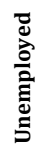 & 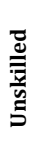 & 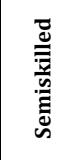 & $\frac{\bar{d}}{\overline{\bar{g}}}$ & 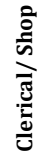 & 离 & 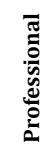 \\
\hline & 3 & 7 & 2 & 6 & 9 & 2 & 0 & 1 & 0 \\
\hline \multirow{2}{*}{$\begin{array}{l}\text { Socio- } \\
\text { economic } \\
\text { status }\end{array}$} & \multicolumn{2}{|c|}{ Upper } & \multicolumn{2}{|c|}{$\begin{array}{c}\text { Upper } \\
\text { Middle }\end{array}$} & \multicolumn{2}{|c|}{\begin{tabular}{l|l} 
Lower \\
Middle
\end{tabular}} & $\begin{array}{l}\text { Upp } \\
\text { Low }\end{array}$ & \multicolumn{2}{|c|}{ Lower } \\
\hline & & ) & \multicolumn{2}{|c|}{3} & \multicolumn{2}{|l|}{17} & 7 & \multicolumn{2}{|c|}{3} \\
\hline \multicolumn{10}{|c|}{ Table 1. Socio-Demographic Profile of the Patients } \\
\hline
\end{tabular}

\begin{tabular}{|c|c|c|}
\hline Final Diagnosis & No. & Percentage \\
\hline Bipolar Affective Disorder & 8 & 26.67 \\
\hline Major Depressive disorder & 1 & 3.33 \\
\hline Brief Psychotic Disorder & 8 & 26.67 \\
\hline Schizophreniform Disorder & 2 & 6.67 \\
\hline Schizophrenia & 4 & 13.33 \\
\hline Unspecified Psychotic Disorder & 2 & 6.67 \\
\hline Organic & 1 & 3.33 \\
\hline Substance & 2 & 6.67 \\
\hline Conversion & 2 & 6.67 \\
\hline Table 2. Final Diagnosis at the Time of Discharge (n=30)
\end{tabular}

\begin{tabular}{|c|c|c|c|c|c|c|}
\hline Variables & Diagnosis & $\mathbf{N}$ & Mean & Std. Deviation & $\mathbf{F}$ & Sig. \\
\hline \multirow{3}{*}{$\begin{array}{l}\text { Duration of } \\
\text { hospital stay }\end{array}$} & Mood disorder & 9 & 12.2 & 4.2 & 3.6 & $0.04^{*}$ \\
\hline & Psychotic disorder & 16 & 21.4 & 11.5 & & \\
\hline & Others & 5 & 13.0 & 4.8 & & \\
\hline \multirow{3}{*}{$\begin{array}{l}\text { lorazepam } \\
\text { dose }\end{array}$} & Mood disorder & 9 & 8.4 & 2.4 & 0.62 & 0.55 \\
\hline & Psychotic disorder & 16 & 9.8 & 4.1 & & \\
\hline & \begin{tabular}{|l|} 
Others \\
\end{tabular} & 5 & 8.0 & 4.0 & & \\
\hline \multirow{3}{*}{$\begin{array}{l}\text { BFCRS Score } \\
\text { on Admission }\end{array}$} & Mood disorder & 9 & 19.3 & 4.8 & 0.64 & 0.53 \\
\hline & \begin{tabular}{|l|} 
Psychotic disorder \\
\end{tabular} & 16 & 22.1 & 7.4 & & \\
\hline & \begin{tabular}{|c|} 
Others \\
\end{tabular} & 5 & 20.0 & 3.7 & & \\
\hline & Total & 30 & & & & \\
\hline \multicolumn{7}{|c|}{${ }^{*}$ The mean difference is significant at the 0.05 level. } \\
\hline \multicolumn{7}{|c|}{$\begin{array}{c}\text { Table 3a. Relationship of Various Psychiatric Diagnoses with } \\
\text { Catatonic Symptoms to Duration of Hospital Stay, Lorazepam } \\
\text { Dosage and BFCRS Scores (One-Way ANOVA) }\end{array}$} \\
\hline
\end{tabular}

\begin{tabular}{|c|c|c|c|}
\hline \multicolumn{4}{|c|}{ Multiple Comparisons } \\
\hline \multicolumn{4}{|c|}{ LSD } \\
\hline Dependent Variable & & & Significance \\
\hline \multirow{6}{*}{$\begin{array}{l}\text { Duration of } \\
\text { hospital stay }\end{array}$} & Mood disorder & Psychotic disorder & \begin{tabular}{|l|}
.022 \\
\end{tabular} \\
\hline & & Others & .879 \\
\hline & Psychotic disorder & Mood disorder & .022 \\
\hline & & Others & .083 \\
\hline & Others & Mood disorder & .879 \\
\hline & & Psychotic disorder & .083 \\
\hline \multicolumn{4}{|c|}{ *The mean difference is significant at the 0.05 level. } \\
\hline
\end{tabular}

There was a statistically significant difference between the groups in terms of the duration of hospital stay determined by one-way ANOVA as shown in Table 3a [F $(2,27)=3.6, p=0.04]$. Regarding the Lorazepam dosage and the BFCRS score, there was no significant difference among the groups. On further analysis in Table $3 \mathrm{~b}$, a post hoc test revealed that the duration of hospital stay was significantly less in mood disorder cases than the psychotic disorders $(\mathrm{p}=$ 0.22). There was no statistically significant difference between those two groups and the other group. Family history of psychiatric illness was present in $20 \%$ of patients with the equal prevalence of affective and psychotic disorders among the family members. The mean duration of catatonic symptoms before clinical examination and consultation at psychiatric hospital was 17.2 days (range: 7-30 days). At the time of discharge, final diagnoses were made as per DSM-5, and $9(30 \%)$ patients were diagnosed with mood disorders (8 with bipolar disorder and 1 with major depressive disorder) and 16 (53.33\%) with psychotic disorders (8 with brief psychotic disorders, 2 with schizophreniform disorders, 4 with schizophrenia and 2 with unspecified psychotic disorders), and rest with others as shown in the table 2 given.

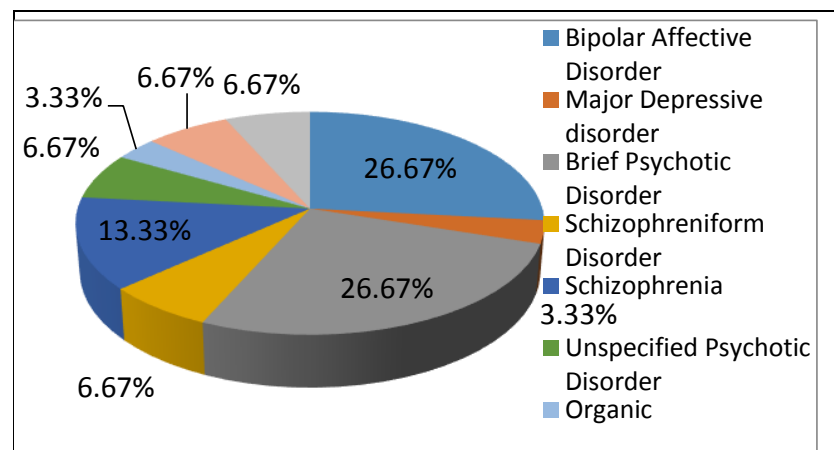

Figure 1. Final Diagnosis at the Time of Discharge $(n=30)$

Among the bipolar disorder cases 4 of them had past history of manic and/or depressive episodes. Prior history of psychotic episodes were present in 2 patients with schizophrenia as well. 4 patients with brief psychotic disorders and 2 with bipolar disorders (single manic episode) presented with initial catatonic presentation along with the respective clinical features before admission. Figure 2 shows the BFCRS score for the assessment of severity of catatonia which was administered to all the cases on the $1^{\text {st }}$ day of admission and at the day of discharge. The mean BFCRS score at admission was $20.93 \pm 6.16$ (range: $11-36$ ). The commonly seen catatonic symptoms which were observed in the study population were mutism, staring, negativism, rigidity, posturing/catalepsy, mannerism, and withdrawal. The other features of catatonia were observed less frequently.

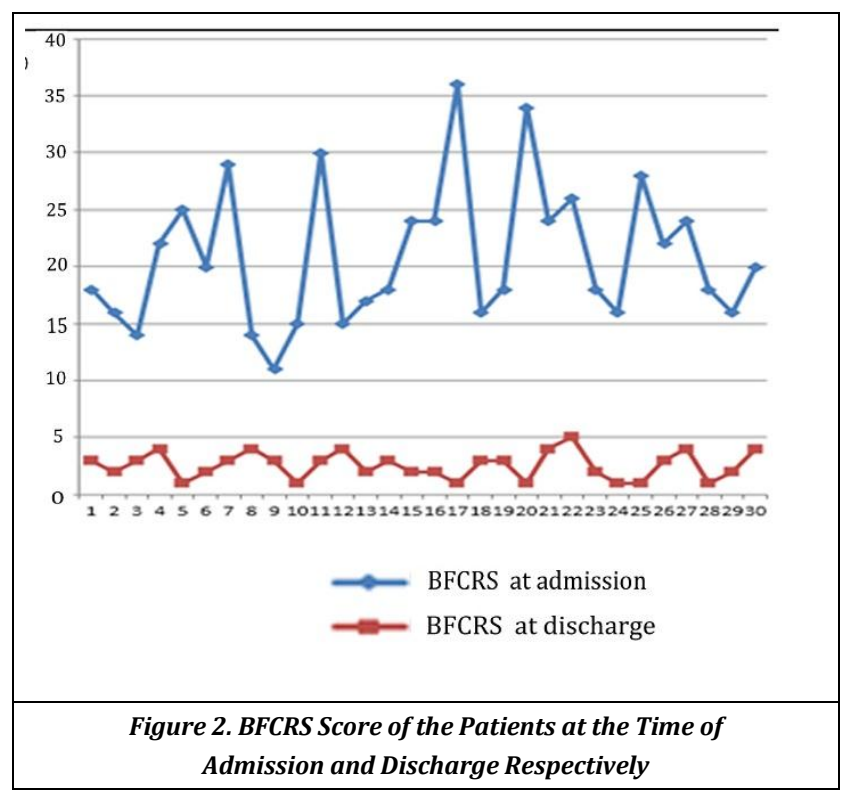


It was seen that $83.33 \%(25$, out of 30$)$ of cases responded well to lorazepam only, whereas the rest $13.33 \%$ (4 out of 30) of patients required ECT after non-response or partial response to lorazepam and the remaining 1 case after diagnosis with catatonia following organic condition were referred to Medicine Department. The days of hospital stay differed with treatment as the mean was found to be $17.23 \pm$ 9.85 (range: 5-44 days). Based on the final diagnosis as per DSM-5, the patients were grouped into broad diagnostic categories as Mood disorders (bipolar disorder and major depressive disorder), Psychotic disorders (brief psychotic disorders, Schizophreniform disorders, schizophrenia \& unspecified psychotic disorders) and Other disorders (organic, substance use and conversion disorders).

The severity of catatonic symptoms as per the BFCRS scores, the maximum dosage of lorazepam received to obtain response and the duration of hospital stay were analysed among these groups using one-way ANOVA. It yielded significant difference only in terms of the days of hospital stay. The results are summarized in Table $3 \mathrm{a} \& 3 \mathrm{~b}$.

\section{DISCUSSION}

The study showed catatonia to be occurring commonly in young, having its onset in twenties (24.63 years) which is in accordance with previous studies.7,10-12 It was found to be common in males, patients with high school education, Hindu religion, housewives and semiskilled workers, patients from mostly rural background, which again matches with other studies from India and represented profile of the majority of the patients. ${ }^{10-12}$ Few patients had a family history of psychiatric illness (20\%) suggestive of possible genetic etiology that is comparable with other studies. ${ }^{11-12}$ Substance use disorder was found to be less common in our patients, compared to the sample from other studies ${ }^{7}$ which might be due to small sample size. This issue requires further in-depth examination with wider population. In the process of diagnosis of catatonia all kind of psychiatric illnesses such as schizophrenia and other psychotic disorders, mood disorders, and organic brain disorders were the final diagnoses which supports the fact that catatonia being an illness of multifactorial aetiology. In our study, psychotic disorders especially brief psychotic disorders were more commonly presented as catatonia as compared to mood disorders. The finding supports few Indian studies done in the similar topic. $7,10-12$

The previous studies have indicated the usefulness of lorazepam in the treatment of catatonia for improving the symptoms, more specifically catatonia following another mental disorder.11,12,18,22 This present study supports the lorazepam challenge notion in the improvement of symptoms of catatonia. Although lorazepam was given to all the patients in the tapering doses, our study findings showed $83 \%$ of the patients were lorazepam responders and other $15 \%$ of the patients after becoming non-responders or partial responder to lorazepam, were treated effectively with ECT. The apparent higher response to lorazepam in this study might be due to different possibilities. Many studies 7,11,12 describing relatively less effectiveness of lorazepam to manage catatonia have applied MECT earlier to attain the response, whereas we delayed the administration of MECT as long as the BFCRS scores were improving along with the patient's functionality. This approach was previously seen with similar results in few earlier studies as well.22-25 Secondly, there is no definite protocol to be used for the dose standardization of lorazepam in the treatment of catatonia till now, which might lead to different response to lorazepam in different studies. ${ }^{7}$

Also, the genetic variation of the population across the countries might have contribution in the difference in treatment response as well. ${ }^{26}$ Further research is needed to validate the claim though. We have assessed whether the severity of catatonia and the dosage and duration of lorazepam to get response has anything to predict from the background psychiatric diagnoses. Regarding background diagnoses, we had mood disorders, psychotic disorders and other categories. In our study there was no significant differences between them in terms of symptom severity or lorazepam dosage. Although it was seen the duration of hospital stay was significantly different in those groups (table 3a \& 3b). This might be due the cause that the psychotic patients, as responding to slow incremental doses of lorazepam as shown in previous studies as well, took longer time of hospital stay. ${ }^{18,27}$ The findings shed light to the importance of further research in this area in India regarding the difference in severity and treatment response in bipolar and psychosis induced catatonic syndrome.

\section{CONCLUSIONS}

This study has come out with very important insights in the clinical profile, diagnostic break ups and treatment response with lorazepam and ECT in catatonic patients following mental disorder. Catatonia is more commonly associated with psychotic disorders than mood disorders in this part of the world.7,10-12 Smaller sample size, shorter duration of study and lack of prospective approach remain as the limitations of the present study. Also, we made the background diagnoses at the time of discharge, the most of which were brief psychotic disorders. Whether their presentation change over the course of time is another area open for further research. Different background diagnoses may dictate the difference in the duration of hospital stay as found in this study.

\section{ACKNOWLEDGEMENT}

We thank our colleagues of the Department of Psychiatry, North Bengal Medical College who provided insight, expertise and great support during this research. 


\section{REFERENCES}

[1] Karl Kahlbaum KL. Die Katatonie Oder Das SpannungIrresein, Eine Klinische Form Psychischer Krankheit. Berlin: Verlag August Hirshwald 1874.

[2] Fink M. Catatonia from its creation to DSM-V: considerations for ICD. Indian J Psychiatry 2011;53(3):214-7.

[3] American Psychiatric Association. Diagnostic and statistical manual of mental disorders (DSM-5). $5^{\text {th }}$ edn. Washington, DC: American Psychiatric Association 2013.

[4] Fink M, Taylor MA. Catatonia: a clinician's guide to diagnosis and treatment. New York: Cambridge University Press 2003:147-69.

[5] Brenner I, Rheuban WJ. The catatonic dilemma. Am J Psychiatry 1978;135(10):1242-3.

[6] Taylor MA, Fink M. Catatonia in psychiatric classification: a home of its own. Am J Psychiatry 2003;160(7):123341.

[7] Dutt A, Grover S, Chakrabarti S, et al. Phenomenology and treatment of catatonia: a descriptive study from North India. Indian J Psychiatry 2011;53(1):36-40.

[8] Jha BK, Reddy GC. Catatonia associated with uraemic encephalopathy. Indian J Psychiatry 1968;10:95-6.

[9] Srivastava A, Gupta A, Murthy P, et al. Catatonia and multiple pressure ulcers: a rare complication in psychiatric setting. Indian J Psychiatry 2009;51(3):2068.

[10] Banerjee A, Sharma LN. Catatonia incidence in acute psychiatric admissions. Indian J Psychiatry 1995;37(1):35-9.

[11] Ramdurg S, Kumar S, Kumar $M$, et al. Catatonia: etiopathological diagnoses and treatment response in a tertiary care setting: a clinical study. Indian Psychiatry J 2013;22(1):32-6.

[12] Swain SP, Behura SS, Dash MK. The phenomenology and treatment response in catatonia: a hospital based descriptive study. Indian J Psychol Med 2017;39(3):3239.

[13] Bush G, Fink M, Petrides G, et al. Catatonia. I. Rating scale and standardized examination. Acta Psychiatr Scand 1996;93(2):129-36.

[14] Sienaert P, Rooseleer J, De Fruyt J. Measuring catatonia: a systematic review of rating scales. J Affect Disord 2011;135(1-3):1-9.
[15] Rooseleer J, Willaert A, Sienaert P. Rating scales for assessing catatonia; which ones are the best? Tijdschr Psychiatr 2011;53(5):287-98.

[16] Fink M, Taylor MA. The catatonia syndrome: forgotten but not gone. Arch Gen Psychiatry 2009;66(11):1173-7.

[17] Rosebush PI, Hildebrand AM, Furlong BG, et al. Catatonic syndrome in a general psychiatric inpatient population: frequency, clinical presentation, and response to lorazepam. J Clin Psychiatry 1990;51(9):357-62.

[18] Ungvari GS, Kau LS, Wai-Kwong T, et al. The pharmacological treatment of catatonia: an overview. Eur Arch Psychiatry Clin Neurosci 2001;251 Suppl 1:131-4.

[19] Malur C, Pasol E, Francis A. ECT for prolonged catatonia. J ECT 2001;17(1):55-9.

[20] Daniels J. Catatonia: clinical aspects and neurobiological correlates. J Neuropsychiatry Clin Neurosci 2009;21(4):371-80.

[21] Carroll BT, Goforth HW, Thomas C, et al. Review of adjunctive glutamate antagonist therapy in the treatment of catatonic syndromes. J Neuropsychiatry Clin Neurosci 2007;19(4):406-12.

[22] Bush G, Fink M, Petrides G, et al. Catatonia, II: Treatment with lorazepam and electroconvulsive therapy. Acta Psychiatr Scand 1996;93(2):137-43.

[23] Girish K, Gill NS. Electroconvulsive therapy in lorazepam non-responsive catatonia. Indian J Psychiatry 2003;45(1):21-5.

[24] Rosebush PI, Mazurek MF. Catatonia and its treatment. Schizophr Bull 2010;36(2):239-42.

[25] Lee JW, Schwartz DL, Hallmayer J. Catatonia in a psychiatric intensive care facility: incidence and response to benzodiazepines. Ann Clin Psychiatry 2000;12(2):89-96.

[26] Rasmussen SA, Mazurek MF, Rosebush PI. Catatonia: our current understanding of its diagnosis, treatment and pathophysiology. World J Psychiatry 2016;6(4):391-8.

[27] Beckmann H, Fritze J, Franzek E. The influence of neuroleptics on specific syndromes and symptoms in schizophrenics with unfavourable long-term course. A 5year follow-up study of 50 chronic schizophrenics. Neuropsychobiology
1992;26(1-2):50-8. 